\title{
Perturbative renormalization in parton distribution functions using improved actions
}

\author{
Mihalis loannou* \\ Univ of Cyprus; Physics Dept \\ E-mail: mike80bi@yahoo.gr \\ Haralambos Panagopoulos \\ Univ of Cyprus; Physics Dept \\ E-mail: haris@ucy.ac.cy
}

\begin{abstract}
We calculate the 1-loop renormalization of a set of extended fermionic bilinears which form a basis corresponding to moments of the parton distribution functions.

We use the overlap action for fermions and Luescher-Weisz (LW) action for gluons.

Our results are presented as a function of the overlap parameter $\rho$ and the parameters entering the LW action.
\end{abstract}

XXIIIrd International Symposium on Lattice Field Theory

25-30 July 2005

Trinity College, Dublin, Ireland

\footnotetext{
* Speaker.
} 


\section{Introduction.}

The description of physics in the Bjorken limit involves the operator product expansion which has the standard form:

$$
J(x) J(0) \sim \sum_{n, i, l} C_{l}^{n, i}\left(x^{2}\right) x^{\mu_{1}} \ldots x^{\mu_{n}} O_{\mu_{1} \ldots \mu_{n}}^{(n, i)}(0)
$$

The forward matrix elements of the local operators $O^{(n, i)}$ appearing in this expansion are directly related to the moments of hadron structure functions. The dominant contribution in the expansion is given by operators whose twist (dimension minus spin) equals two, which in the flavor non-singlet case means the symmetric traceless operators[ []

$$
\begin{gathered}
O_{\mu \mu_{1} \ldots \mu_{n}}=\bar{\psi} \gamma_{\{\mu} D_{\mu_{1}} \ldots D_{\left.\mu_{n}\right\}} \frac{\lambda^{a}}{2} \psi \\
O_{\mu \mu_{1} \ldots \mu_{n}}^{(5)}=\bar{\psi} \gamma_{\{\mu} \gamma_{5} D_{\mu_{1} \ldots D_{\left.\mu_{n}\right\}}} \frac{\lambda^{a}}{2} \psi
\end{gathered}
$$

where $\lambda^{a}$ are flavor matrices. In this work we compute the renormalization factors of the following quantities on the lattice.

a) Self-energy.

$$
S_{N}^{-1}=i \gamma_{\mu} P_{\mu}\left(1-\frac{g^{2} C_{F}}{16 \pi^{2}} \Sigma_{1}\right)
$$

b) Quark bilinears.

$$
O_{X}=\bar{\psi}(x) \Gamma^{X} \psi(x)
$$

c) Operators which measure the first moment of quark momentum distributions.

$$
\begin{gathered}
O_{V_{2}}=\bar{\psi} \gamma_{\{1} D_{4\}} \psi \\
O_{V_{2}}^{\prime}=\bar{\psi} \gamma_{4} D_{4} \psi-\frac{1}{3} \sum_{i=1}^{3} \bar{\psi} \gamma_{i} D_{i} \psi
\end{gathered}
$$

d) Operators which measure the first moment of quark helicity distributions.

$$
\begin{gathered}
O_{A_{2}}=\bar{\psi} \gamma_{\{1} \gamma_{5} D_{4\}} \psi \\
O_{A_{2}}^{\prime}=\bar{\psi} \gamma_{4} \gamma_{5} D_{4} \psi-\frac{1}{3} \sum_{i=1}^{3} \bar{\psi} \gamma_{i} \gamma_{5} D_{i} \psi
\end{gathered}
$$

Work is in progress using operators which measure the second moment of quark distributions. A longer write-up of our results can be found in our Ref.[2]. 


\section{Calculational details.}

We denote the lattice action by

$$
S=S_{G}+S_{F}
$$

where $S_{G}$ is the gluon action, and $S_{F}$ is the fermion action. The gluon action we consider is written in standard notation:

$$
S_{g l}=-\frac{1}{g^{2}}\left(c_{0} \sum_{\text {plaquette }} \operatorname{Tr} U_{p l}+c_{1} \sum_{\text {rectangle }} \operatorname{Tr} U_{\text {rtg }}+c_{2} \sum_{\text {chair }} \operatorname{Tr} U_{\text {chr }}+c_{3} \sum_{\text {parallelogram }} \operatorname{Tr} U_{\text {plg }}\right)
$$

where $U_{p l}$ is the standard plaquette, while the remaining U's cover all possible closed loops containing up to six links. The coefficients $c_{0}, c_{1}, c_{2}, c_{3}$ satisfy the normalization condition:

$$
c_{0}+8 c_{1}+16 c_{2}+8 c_{3}=1
$$

As in Ref.[3], we have used the values of $c_{0}, c_{1}, c_{2}, c_{3}$, shown in Table I, where "Plaquette" is the standard Wilson action for gluons, "Symanzik" is the tree-level improved action [ $₫$ ] and TILW is the tadpole improved Luescher-Weisz action [5, 6].

\begin{tabular}{|c|l|l|l|l|}
\multicolumn{1}{c}{ action } & \multicolumn{1}{c}{$c_{0}$} & \multicolumn{1}{c}{$c_{1}$} & \multicolumn{1}{c|}{$c_{2}$} & \multicolumn{1}{c|}{$c_{3}$} \\
\hline Plaquette & 1.0 & 0.0 & 0.0 & 0.0 \\
Symanzik & 1.66666 & -0.083333 & 0.0 & 0.0 \\
TILW1 $\beta=8.60$ & 2.31681 & -0.151791 & 0.0 & -00128098 \\
TILW2 $\beta=8.45$ & 2.34602 & -0.154846 & 0.0 & -0.0134070 \\
TILW3 $\beta=8.30$ & 2.38698 & -0.159128 & 0.0 & -0.0142442 \\
TILW4 $\beta=8.20$ & 2.41278 & -0.161827 & 0.0 & -0.0147710 \\
TILW5 $\beta=8.10$ & 2.44654 & -0.165353 & 0.0 & -0.0154645 \\
TILW6 $\beta=8.00$ & 2.48917 & -0.169805 & 0.0 & -0.0163414 \\
Iwasaki [7] & 3.648 & -0.331 & 0.0 & 0.0 \\
DBW2 [8] & 12.2688 & -1.4086 & 0.0 & 0.0 \\
\hline
\end{tabular}

Table 1: The values of coeffi cients $q_{0}, c_{1}, c_{2}, c_{3}$

The action for massless overlap fermions is given by [0]

$$
S_{f}=\sum_{f} \sum_{x, y} \bar{\psi}_{x}^{f} D_{N}(x, y) \psi_{y}^{f}
$$

with

$$
D_{N}=\rho\left[1+X\left(X^{\dagger} X\right)^{-\frac{1}{2}}\right]
$$

and: $X=D_{w}-\rho$. Here $D_{w}$ is the massless Wilson-Dirac operator with $r=1$, and $\rho$ is a free parameter whose value must be in the range $0<\rho<2$ in order to guarantee the correct pole structure of $D_{N}$. 


\section{Calculations and Results.}

\section{Self energy}

Let us consider the massless quark propagator $S_{N}$ first. The inverse of $S_{N}$ can be written

$$
S_{N}^{-1}=i \gamma_{\mu} p_{\mu}\left(1-\frac{g^{2} C_{F}}{16 \pi^{2}} \Sigma_{1}\right)
$$

with $C_{F}=\left(N^{2}-1\right) / 2 N$, and $\Sigma_{1}(a, p)=\log \left(a^{2} p^{2}\right)+b_{\Sigma}$ (Feynman gauge). Our results for $b_{\Sigma}$ are shown in Fig.1.

\section{Quark bilinears}

Let us consider local operators of the form

$$
O_{X}=\bar{\psi}(x) \gamma^{X} \psi(x)
$$

with $\Gamma^{S}=1, \Gamma^{P}=\gamma_{5}, \Gamma^{V}=\gamma_{\mu}, \Gamma^{A}=\gamma_{\mu} \gamma_{5}, \Gamma^{T}=\sigma_{\mu \nu} \gamma_{5}$, i.e. $X=S, P, V, A$ and $T$. We denote the amputated Green's function of the operator $O_{X}$ by $\Lambda^{X}$. The final results are (in Feynman gauge):

$$
\begin{gathered}
\Lambda^{S, P}=\left\{1, \gamma_{5}\right\}+\frac{g^{2} C_{F}}{16 \pi^{2}}\left[-4 \log \left(a^{2} p^{2}\right)+b_{S, P}\right]\left\{1, \gamma_{5}\right\} \\
\Lambda_{\mu}^{V, A}=\left\{\gamma_{\mu}, \gamma_{\mu} \gamma_{5}\right\}+\frac{g^{2} C_{F}}{16 \pi^{2}}\left[-\gamma_{\mu}\left(\log \left(a^{2} p^{2}\right)+b_{V, A}\right)+2 \frac{p_{\mu} \not p}{p^{2}}\right]\left\{1, \gamma_{5}\right\} \\
\Lambda_{\mu, v}^{T}=\sigma_{\mu, \nu} \gamma_{5}+\frac{g^{2} C_{F}}{16 \pi^{2}} b_{T} \sigma_{\mu, \nu} \gamma_{5}
\end{gathered}
$$

Where $b_{S}=b_{P}$ and $b_{V}=b_{A}$. Only one diagram ("Vertex") contributes to $\Lambda^{X}$.

Our results for $b_{\Sigma}, b_{S, P}, b_{V, A}$ and $b_{T}$ provide a cross check and an extension of results appearing in Refs. [10, 3]. They are shown in Figures 1, 2, 3, 4 for typical values of the overlap parameter $\rho$.

Tables of numerical results are presented in our ref [2]. In all cases which can be compared, our results agree with those of Refs.[10, 3]. There is one exception, regarding the values of $b_{T}$ for cases other than the standard plaquette $b_{T}^{\text {plaq }}:$ In these cases, our results for $b_{T}-b_{T}^{\text {plaq }}$ have the opposite sign compared to Ref. [3].

\section{First moment of quark distributions.}

We have calculated the 1-loop renormalization coefficients of the operators $O_{V_{2}}, O_{V_{2}}^{\prime}$ which are the symmetric off-diagonal, and the traceless diagonal parts, respectively, of the operator:

$$
O_{\mu, v}=\bar{\psi} \gamma_{\{\mu} D_{v\}} \psi
$$

The axial counterparts $O_{A_{2}}, O_{A_{2}}^{\prime}$ of the above operators renormalize in the same way. Using the notation of Ref.[11], we find for the amputated Green's function of $O_{\mu, v}$ in the Feynman gauge:

$$
\Lambda_{\mu, v}(a, p)=\gamma_{\{\mu} p_{v\}}+\frac{g^{2} C_{F}}{16 \pi^{2}}\left[\left(\frac{5}{3} \log \left(a^{2} p^{2}\right)+b\right) \gamma_{\{\mu} p_{v\}}+b^{\prime} \delta_{\mu v} \gamma_{v} p_{v}-\frac{4}{3} \frac{p_{\mu} p_{v}}{p^{2}} \not p\right]
$$

The quantities $b, b^{\prime}$ correspond to $\left(b_{1}+b_{2}\right), b_{4}$ of Ref.[3], respectively. A total of four diagrams ("Tadpole", "Vertex", left and right "Sails") contribute in this case. The rational coefficients $5 / 3$ and $-4 / 3$ in Eq. (3.7) check with those of Ref.[11]. The values of $b$ and $b^{\prime}$ are shown in Figures 5 and 6. 


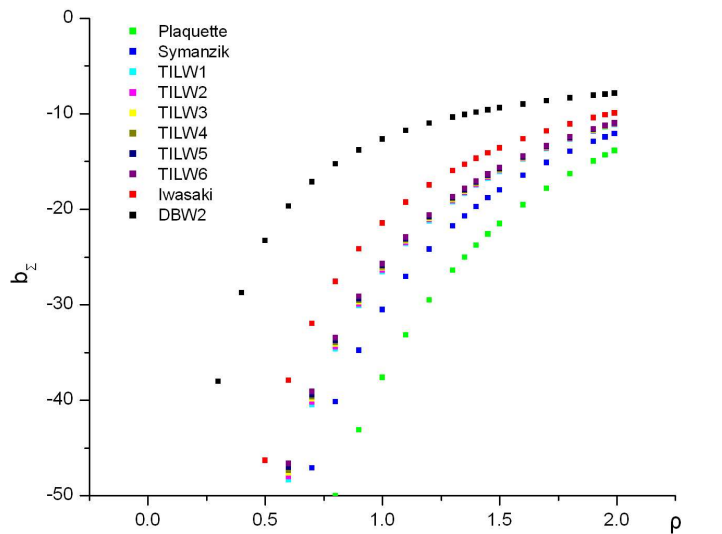

$b_{\Sigma}$ as a function of the overlap parameter $\rho$, shown on a different scale.

Figure 1

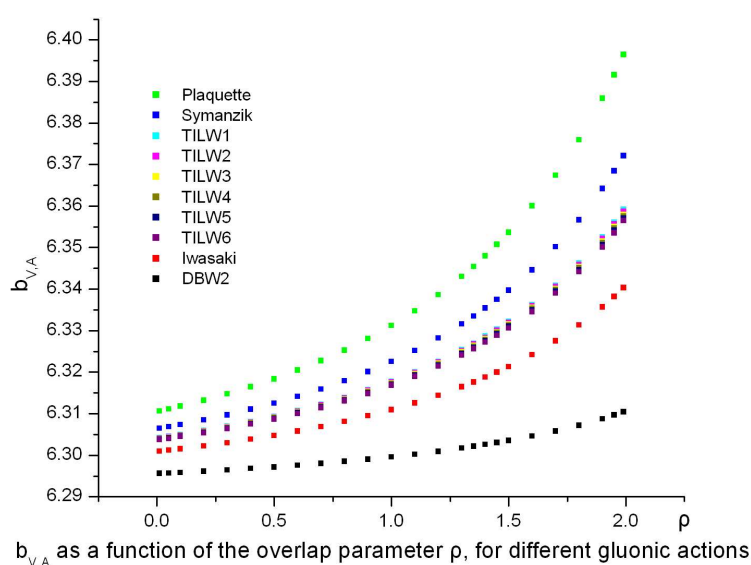

Figure 3

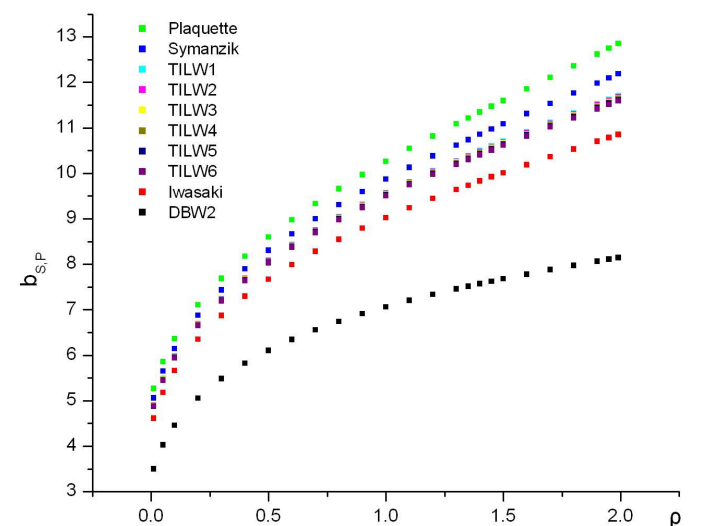

$b_{S, P}$ as a function of the overlap parameter $p$, for different gluonic actions.

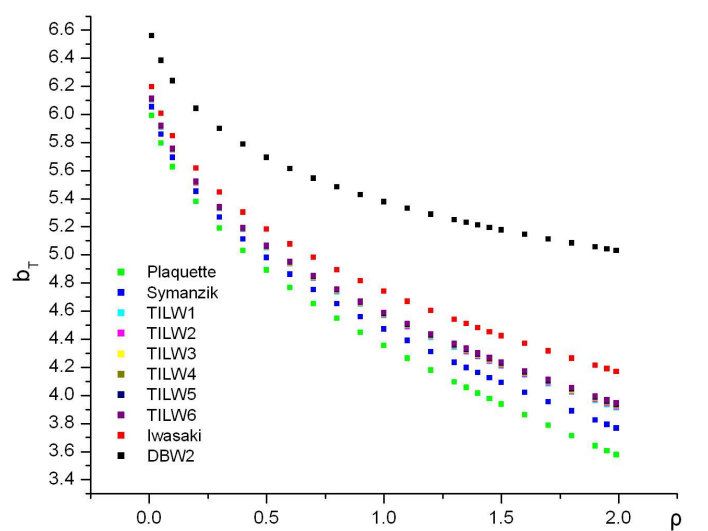

$b_{T}$ as a function of the overlap parameter $\rho$, for different gluonic actions.

\section{References}

[1] S. Capitani, Perturbative renormalization of the first two moments of non-singlet quark distributions with overlap fermions, Nucl.Phys. B592 (2001) 183.

[2] M. Ioannou and H. Panagopoulos, Moments of quark distributions using overlap and Symanzik improved actions, in preparation.

[3] R. Horsley, H. Perlt, P. E. L. Rakow, G. Schierholz and A. Schiller, One-loop renormalization of quark bilinears for overlap fermions with improved gauge actions, Nucl. Phys. B693 (2004) 3-35.

[4] K. Symanzik, Continuum limit and improved action in lattice theories. 1. Principles and phi**4 theory, Nucl. Phys. B226 (1983) 187. 


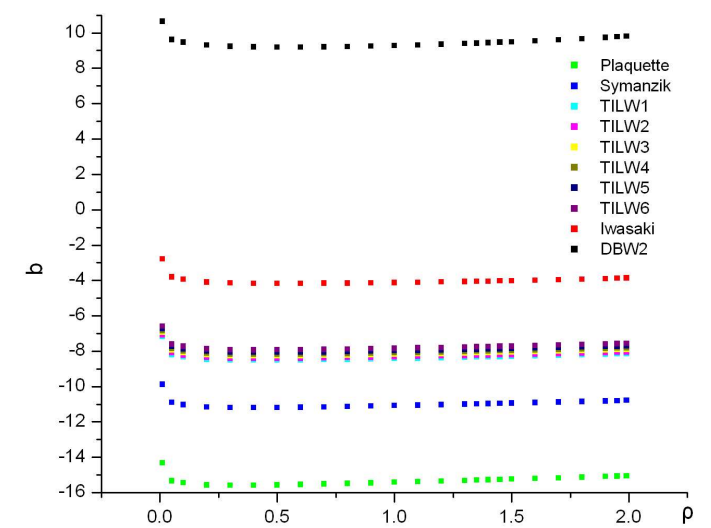

b as a function of the overlap parameter $\rho$, for different gluonic actions.

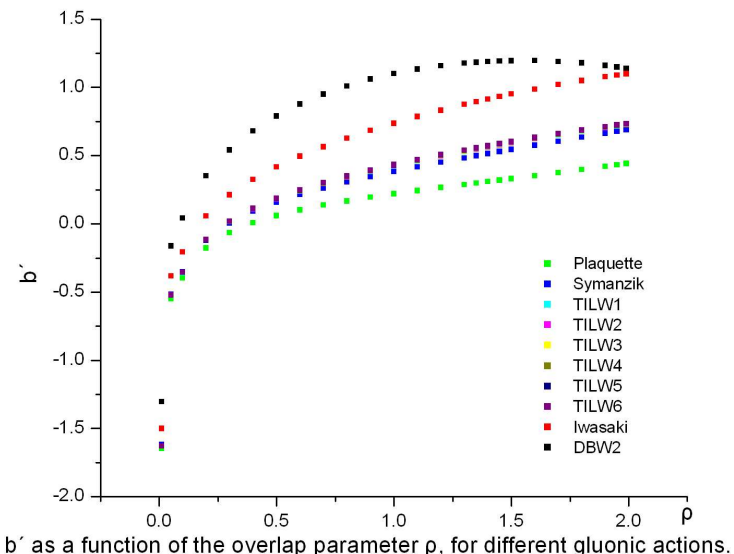

Figure 6

[5] M. Lüscher and P. Weisz, On-shell improved lattice gauge theories, Commun. Math. Phys. 97 (1985) 59 [Erratum-ibid. 98 (1985) 433].

[6] M. G. Alford, W. Dimm, G. P. Lepage, G. Hockney and P. B. Mackenzie, QCD on small computers, Phys. Lett. B361 (1995) 87.

[7] Y. Iwasaki, Renormalization group analysis of lattice theories and improved lattice action. 2. Four-dimensional nonabelian SU(N) gauge model, UTHEP-118 (1983).

[8] T. Takaishi, Heavy quark potential and effective actions on blocked configurations, Phys. Rev. D54 (1996) 1050.

[9] H. Neuberger, Exactly massless quarks on the lattice, Phys. Lett. B417 (1998) 141; More about exactly massless quarks on the lattice, Phys. Lett. B427 (1998) 353.

[10] C. Alexandrou, E. Follana, H. Panagopoulos and E. Vicari, Lattice QCD with the Overlap-Dirac Operator: Its $\Lambda$ Parameter, and One-loop Renormalization of Fermionic Currents, Nucl. Phys. B580 (2000) 394.

[11] R. Horsley, H. Perlt, P. E. L. Rakow, G. Schierholz and A. Schiller, Renormalization of one-link quark operators for overlap fermions with Lüscher-Weisz gauge action, hep -lat/0505015 (2005). 